\title{
The Generalized Inverse of Atanassov's Intuitionistic Fuzzy Matrices
}

\author{
Rajkumar Pradhan and Madhumangal Pal \\ Department of Applied Mathematics with Oceanology and Computer Programming \\ Vidyasagar University, Midnapore-721102, India. \\ E-mail: \{math.rajkumar,mmpalvu\}@gmail.com
}

Received 8 July 2013

Accepted 4 February 2014

\begin{abstract}
In this paper, a method is presented to find generalized inverse (g-inverse) of Atanassov's intuitionistic fuzzy matrix (AIFM). Also, the idea of standard basis for Atanassov's intuitionistic fuzzy vectors is introduced. Some results regarding the g-inverse of AIFM are studied. An application of g-inverse is provided at the end of the paper.
\end{abstract}

Keywords: Atanassov's intuitionistic fuzzy set, intuitionistic fuzzy matrix, generalized inverse (g-inverse), minus ordering.

\section{Introduction}

The generalized inverse ( $g$-inverse) is a generalization of the inverse of matrix to the case of a singular or a non-square matrix. Like the inverse of matrices, the $g$-inverse of matrices is important in a variety of applications such as control, robotics, signal processing and associative memories.

Initially, fuzzy set theory was proposed by Zadeh $^{1}$ as a means of representing mathematically any imprecise or vague system of information in the real world. In fuzzy set theory, there were no scope to think about the hesitation in the membership degrees which is arise in various real life situations. This situation is overcome by invention of Atanassov's intuitionistic fuzzy sets (AIFSs) by Atanassov ${ }^{2}$. Here it is possible to model hesitation and uncertainty by using an additional degree. The fuzzy matrix have been proposed to represent fuzzy relation in a system based on fuzzy sets theory. Several authors presented a number of results on fuzzy matrices ${ }^{3,4,5,6,7,8}$. Pal and Shyamal ${ }^{9,10}$ shown several properties on fuzzy matrices and interval-valued fuzzy matrices. Dehghan et al. ${ }^{11}$ give two ideas for finding the inverse of a fuzzy matrix, 'scenario-based' and 'arithmetic-based'. In the first idea, they will consider a real matrix $A$, which is derived from a fuzzy matrix $\tilde{A}$ and define the fuzzy inverse with respect to scenarios. In the second idea, they try to find a fuzzy matrix $\tilde{B}$ as the inverse of fuzzy matrix $\tilde{A}$, such that $\tilde{A} \otimes \tilde{B}=\tilde{I}$, where $\tilde{I}$ is the fuzzy identity matrix. Nanda $^{12}$ studied intuitionistic fuzzy relations over AIFSs. Using the concept of AIFSs, first time $\mathrm{Pal}^{13}$ introduced Atanassov's intuitionistic fuzzy determinant (AIFD) and Atanassov's intuitionistic fuzzy matrices (AIFMs). Using the idea of AIFSs and AIFD Pal et al. ${ }^{14,15,16}$ studied several properties of AIFMs. Latter on Sriram $^{17}$ studied semi ring of AIFMs. Mondal and Samanta ${ }^{18}$ gives the idea of Atanassov's generalized intuitionistic fuzzy sets (AGIFSs). Using the concept of AGIFSs, Bhowmik and $\mathrm{Pal}^{19}$ introduced AGIFMs with some properties and studied on Atanassov's intuitionistic circulant fuzzy matrix. Perhaps first time Bhowmik et al. ${ }^{20,21}$ introduce the definition of generalized Atanassov's interval-valued intuitionistic fuzzy sets and studied several properties on it. Khan and $\mathrm{Pal}^{22}$ introduced the concept of generalized inverse for AIFMs, minus ordering and shown many results on it.

The authors of ${ }^{3,4}$, defined generalized inverse of fuzzy matrix and based on this definitions they have produced several results. To the best of our knowledge no algorithms are available to determine the generalized inverse of fuzzy matrix. In this paper, we studied generalized inverse of AIFM and presented a simple algorithm to evaluate it. A rectangular system of Atanassov's intuitionistic fuzzy relational equations has been solved using $g$-inverse of AIFM. 


\section{Preliminary Definitions}

Here we recall some preliminary definitions regarding the topic.

By a fuzzy matrix, we mean a matrix over a fuzzy algebra. A fuzzy algebra is a mathematical system $(F,+,$.$) with two binary operations addition (+)$ and multiplication(.) defined on a set $F$ satisfying the following properties:

(P1) Idempotence $a+a=a, a . a=a$

(P2) Commutativity $a+b=b+a, a \cdot b=b \cdot a$

(P3) Associativity $a+(b+c)=(a+b)+c$,

a. $(b . c)=(a . b) \cdot c$

(P4) Absorption $a+a \cdot b=a, a \cdot(a+b)=a$

(P5) Distributivity $a .(b+c)=(a \cdot b)+(a . c)$, $a+(b . c)=(a+b) \cdot(a+c)$

(P6) Universalbounds $a+0=a, a+1=1 ; a .0=0, a .1=a$.

A fuzzy matrix can be interpreted as a binary fuzzy relation, which is defined as follows.

\section{Definition 1. (Fuzzy matrix)}

A fuzzy matrix (FM) of order $m \times n$ is defined as $A=\left\langle a_{i j}, a_{i j \mu}\right\rangle$ where $a_{i j \mu}$ is the membership value of the $i j$-th element in $A$. Let $F_{m \times n}$ denotes the set of all fuzzy matrices of order $m \times n$. If $m=n$, in short, we write $F_{n}$, the set of all square FMs of order $n$.

Fuzzy set was not enough to study the hesitation about the membership degree of an element in a set. For dealing this situation Atanassov [2] introduced intuitionistic fuzzy sets, which is defined below.

\section{Definition 2. (Atanassov's intuitionistic fuzzy set)}

An AIFS $A$ is defined as an object of the form $A=\left\{\left\langle x, \mu_{A}(x), v_{A}(x)\right\rangle / x \in X\right\}$ where the function $\mu_{A}: X \rightarrow[0,1]$ and $v_{A}: X \rightarrow[0,1]$ define the degree of membership and degree of non-membership of an element $x \in X \quad$ respectively and $0 \leq \mu_{A}(x)+v_{A}(x) \leq 1$, for every $x \in X$.

The value of $\pi_{A}(x)=1-\mu_{A}(x)-v_{A}(x)$ is called the degree of non-determinacy (or hesitation) of the element $x \in X$ to the AIFS $A$.

Let us define $\langle F\rangle=\{\langle x, y\rangle: x, y \in[0,1]$ and $0 \leq x+y \leq 1\}$.

Definition 3. (Atanassov's intuitionistic fuzzy atrices)
An Atanassov's intuitionistic fuzzy matrix (AIFM) $A$ of order $m \times n$ is defined as $A=\left[x_{i j},\left\langle a_{i j \mu}, a_{i j v}\right\rangle\right]_{m \times n}$ where $a_{i j \mu}, a_{i j v}$ are called membership and non-membership values of $x_{i j}$ in $A$, which maintains the condition $0 \leq a_{i j \mu}+a_{i j \nu} \leq 1$. For simplicity, we write $A=\left[a_{i j}\right]_{m \times n} \quad$ where $a_{i j}=\left\langle a_{i j \mu}, a_{i j \nu}\right\rangle$.

In arithmetic operations, only the values of $a_{i j \mu}$ and $a_{i j v}$ are considered so from here we only consider the values of $a_{i j}=\left\langle a_{i j \mu}, a_{i j \nu}\right\rangle$. All elements of an AIFM are the members of $\langle F\rangle$.

To study several properties of AIFM, we define two algebraic operations, such as componentwise addition and multiplication of AIFMs as follows.

Definition 4. Let $a$ and $b$ be two elements of an AIFM $X$ such that $a=\left\langle a_{i j \mu}, a_{i j v}\right\rangle, \quad b=\left\langle b_{i j \mu}, b_{i j v}\right\rangle$, then componentwise addition and multiplication are defined as, $a+b=\left\langle\max \left\{a_{i j \mu}, b_{i j \mu}\right\}, \min \left\{a_{i j v}, b_{i j \nu}\right\}\right\rangle$ and $a * b=\left\langle\min \left\{a_{i j \mu}, b_{i j \mu}\right\}, \max \left\{a_{i j v}, b_{i j v}\right\}\right\rangle$.

Definition 5. Let $A=\left(\left\langle a_{i j \mu}, a_{i j \nu}\right\rangle\right) \in F_{m \times n} \quad$ and $B=\left(\left\langle b_{i j \mu}, b_{i j \nu}\right\rangle\right) \in F_{m \times n}$, then the matrix addition is given by

$A+B=\left(\left\langle\max \left\{a_{i j \mu}, b_{i j \mu}\right\}, \min \left\{a_{i j \nu}, b_{i j v}\right\}\right\rangle\right) \in F_{m \times n}$ and the product of $A=\left(\left\langle a_{i j \mu}, a_{i j \nu}\right\rangle\right) \in F_{m \times p}$ and $B=\left(\left\langle b_{i j \mu}, b_{i j \nu}\right\rangle\right) \in F_{p \times n} \quad$ is given by, $A B=\left(\left\langle\max _{k}\left\{\min \left(a_{i k \mu}, b_{k j \mu}\right)\right\}, \min _{k}\left\{\max \left(a_{i k v}, b_{k j v}\right)\right\}\right\rangle\right)$, where $k=1,2, \ldots p, i=1,2, \ldots m$ and $j=1,2, \ldots n$.

In our further work we use some special types of AIFMs, which are defined as follows.

\section{Definition 6. (Null AIFM)}

An AIFM is null if all elements of it are zero, that is, all elements are $\langle 0,0\rangle$ and it is denoted by $I_{\langle 0,0\rangle}$. An AIFM is zero if all elements are $\langle 0,1\rangle$ and it is denoted by $\mathbf{O}$.

Definition 7. (Identity AIFM) 
An intuitionistic fuzzy identity matrix of order $n \times n$ is denoted by $I_{n}$ and is defined by $\left\langle\delta_{i j \mu}, \delta_{i j \nu}\right\rangle$ where, $\left\{\begin{array}{l}\delta_{i j \mu}=1, \delta_{i j v}=0 \quad \text { if } \quad i=j \\ \delta_{i j \mu}=0, \delta_{i j v}=1 \quad \text { if } \quad i \neq j\end{array}\right.$.

The universal matrix of order $m \times n$ is denoted by $J$ and all the elements of it are $\langle 1,0\rangle$.

\section{Definition 8. (Multiplication by scalar)}

Let $A=\left(\left\langle a_{i j \mu}, a_{i j v}\right\rangle\right) \in F_{m \times n}$ and $c \in F$ such that $0 \leq c \leq 1$, then the scalar multiplication is defined

$c A=\left(\left\langle\min \left\{c, a_{i j \mu}\right\}, \max \left\{(1-c), a_{i j v}\right\}\right\rangle\right) \in F_{m \times n}$.

Comparison between AIFMs have an important role in our work, which is defined below.

\section{Definition 9. (Dominance of AIFM)}

Let $A, B \in F_{m \times n}$ such that $A=\left(\left\langle a_{i j \mu}, a_{i j v}\right\rangle\right)$ and $B=\left(\left\langle b_{i j \mu}, b_{i j v}\right\rangle\right)$, then we write $A \leq B$ if, $a_{i j \mu} \leq b_{i j \mu}$ and $a_{i j v} \geq b_{i j v}$ for all $i, j$, and we say that $A$ is dominated by $B$ or $B$ dominates $A . A$ and $B$ are said to be comparable, if either $A \leq B$ or $B \leq A$.

The relation ' $\leq$ ' is a partial order relation and $(F, \leq)$ forms a distributive lattice ${ }^{23}$.

\section{Definition 10. (Transpose)}

The transpose $A^{T}$ of an AIFM $A=\left[a_{i j}\right]_{m \times n}$ is defined as $A^{T}=\left[a_{j i}\right]_{n \times m}$ where $a_{j i}=\left\langle a_{j i \mu}, a_{j i v}\right\rangle$. below.

Now, we define a particular type of AIFM

\section{Definition 11. (Intuitionistic fuzzy permutation matrix)}

A square AIFM is called Atanassov's intuitionistic fuzzy permutation matrix (AIFPM), if every row and column contains exactly one $\langle 1,0\rangle$ and all other entries are $\langle 0,1\rangle$.

Definition 12. An AIFM $A \in F_{n}$ is said to be invertible if and only if there exists another AIFM $B \in F_{n}$ such that $A B=B A=I_{n}$.
Let $A=\left(\left\langle a_{i j \mu}, a_{i j v}\right\rangle\right) \in F_{n}$ be invertible, then there exists $B=\left(\left\langle b_{i j \mu}, b_{i j v}\right\rangle\right) \in F_{n}$ such that $A B=B A=I_{n}$. By max-min composition, $\max _{k}\left\{\min \left(a_{i k \mu}, b_{k j \mu}\right)\right\}=0, \min _{k}\left\{\max \left(a_{i k v}, b_{k j v}\right)\right\}=1$ for $i \neq j$ and $\max _{k}\left\{\min \left(a_{i k \mu}, b_{k i \mu}\right)\right\}=1, \min _{k}\left\{\max \left(a_{i k v}, b_{k i v}\right)\right\}=0$ for each $i$.

From (1) we get, $a_{i k \mu}=0$ or $b_{k j \mu}=0$ or both $a_{i k \mu}=b_{k j \mu}=0$ and $a_{i k v}=1$ or $b_{k j v}=1$ or both $a_{i k v}=b_{k j v}=1$ for all $i \neq j$ and for all $k$.

From (2) we get,

$a_{i k \mu}=1$ or $b_{k i \mu}=1$ or both $a_{i k \mu}=b_{k i \mu}=1$ and

$a_{i k v}=0$ or $b_{k i v}=0$ or both $a_{i k v}=b_{k i v}=0$ for at least one $k$ and for each $i$.

Suppose (4) holds for $k=m$ (say), that is, $a_{i m \mu}=b_{i m \mu}=1$ and $a_{i m \nu}=b_{i m v}=0$, then from (3), $a_{j m \mu}=b_{m j \mu}=0$ and $a_{j m \nu}=b_{m j v}=1$ for all $i \neq j$.

Therefore the $r$ th row of $B$ has exactly one $\langle 1,0\rangle$ and remaining all entries are $\langle 0,1\rangle$ and the $r$ th column of $A$ has exactly one $\langle 1,0\rangle$ and remaining all entries are $\langle 0,1\rangle$. Again $B=A^{T}$. Therefore $A$ is permutation matrix and its transpose is the unique inverse of $A$.

Let $P_{n}$ be the set of all AIFMs in $F_{n}$. If $A \in P_{n}$, then $A A^{T}=A^{T} A=I_{n}$. As fuzzy matrices, the AIFMs are the only invertible matrices and then $P^{-1}=P^{T}$ where $P$ is an AIFPM.

\section{Atanassov's Intuitionistic Fuzzy Vectors}

Let $V_{n}$ be the set of all $n$-tuples $\left(\left\langle x_{1 \mu}, x_{1 v}\right\rangle,\left\langle x_{2 \mu}, x_{2 v}\right\rangle, \ldots,\left\langle x_{n \mu}, x_{n v}\right\rangle\right)$ over $\langle F\rangle$. An element of $V_{n}$ is called an Atanassov's intuitionistic fuzzy vector (AIFV) of dimension $n$, where $x_{i \mu}$ and 
$x_{i v}$ are the membership and non-membership values of the component $x_{i}$.

The operations addition (+) and multiplication (.) are defined on $V_{n}$ as follows:

Let $x=\left(\left\langle x_{1 \mu}, x_{1 v}\right\rangle,\left\langle x_{2 \mu}, x_{2 v}\right\rangle, \ldots,\left\langle x_{n \mu}, x_{n v}\right\rangle\right)$ and $y=\left(\left\langle y_{1 \mu}, y_{1 v}\right\rangle,\left\langle y_{2 \mu}, y_{2 v}\right\rangle, \ldots,\left\langle y_{n \mu}, y_{n v}\right\rangle\right)$ be two

IFVs in $V_{n}$, then

$x+y=\left(\left\langle\max \left(x_{1 \mu}, y_{1 \mu}\right), \min \left(x_{1 v}, y_{1 v}\right)\right\rangle\right.$,

$\left\langle\max \left(x_{2 \mu}, y_{2 \mu}\right), \min \left(x_{2 v}, y_{2 v}\right)\right\rangle$,

$\left.\ldots,\left\langle\max \left(x_{n \mu}, y_{n \mu}\right), \min \left(x_{n v}, y_{n v}\right)\right\rangle\right)$

and

$a x=\left(\left\langle\min \left(a, x_{1 \mu}\right), \max \left(1-a, x_{1 v}\right)\right\rangle\right.$,

$\left\langle\min \left(a, x_{2 \mu}\right), \max \left(1-a, x_{2 v}\right)\right\rangle$,

$\left.\ldots,\left\langle\min \left(a, x_{n \mu}\right), \max \left(1-a, x_{n v}\right)\right\rangle\right)$

for $a \in[0,1]$.

The system $V_{n}$ together with these operations of componentwise addition and multiplication forms Atanassov's intuitionistic fuzzy vectors space (AIFVS).

\section{Definition 13. (Linear combination of AIFVs)}

Let $S=\left\{a_{1}, a_{2}, \ldots, a_{p}\right\}$ be a set of AIFVs of dimension $n$. The linear combination of elements of the set $S$ is a finite sum $\sum_{i=1}^{p} c_{i} a_{i}$ where $a_{i} \in S$ and $c_{i} \in[0,1]$. The set of all linear combinations of the elements of $S$ is called the span of $S$, denoted by $\langle S\rangle$.

An example of $V_{3}$ and its spanning set is given below.

Example 1. Let $S=\left\{a_{1}, a_{2}, a_{3}\right\}$ be a subset of $V_{3}$,

Where,

$a_{1}=(\langle 0.8,0.2\rangle,\langle 0.6,0.3\rangle,\langle 0.4,0.3\rangle)$,

$a_{2}=(\langle 0.5,0.3\rangle,\langle 0.5,0.1\rangle,\langle 0.4,0.2\rangle)$

and $a_{3}=(\langle 0.7,0.3\rangle,\langle 0.7,0.2\rangle,\langle 0.9,0.1\rangle)$.

Then,

$$
\begin{aligned}
& \langle S\rangle=\left\{c_{1}(\langle 0.8,0.2\rangle,\langle 0.6,0.3\rangle,\langle 0.4,0.3\rangle)+\right. \\
& c_{2}(\langle 0.5,0.3\rangle,\langle 0.5,0.1\rangle,\langle 0.4,0.2\rangle)+ \\
& \left.c_{3}(\langle 0.7,0.3\rangle,\langle 0.7,0.2\rangle,\langle 0.9,0.1\rangle)\right\} .
\end{aligned}
$$

\section{Definition 14. (Dependence of AIFVs)}

A set $S$ of AIFVs is independent if and only if each element of $S$ can not be expressed as a linear combination of other elements of $S$, that is, no element $s \in S$ is a linear combination of $S \backslash\{s\}$.

A vector $\alpha$ may be expressed by some other vectors. If it is possible then the vector $\alpha$ is called dependent otherwise it is called independent. These terminologies are similar to classical vectors.

The examples of independent and dependent set of vectors are given below.

Example 2 . Let $S=\left\{a_{1}, a_{2}, a_{3}\right\}$ be a subset of $V_{3}$, where

$a_{1}=(\langle 0.8,0.2\rangle,\langle 0.6,0.3\rangle,\langle 0.4,0.3\rangle)$,

$a_{2}=(\langle 0.5,0.3\rangle,\langle 0.5,0.1\rangle,\langle 0.4,0.2\rangle)$

and $a_{3}=(\langle 0.7,0.3\rangle,\langle 0.7,0.2\rangle,\langle 0.9,0.1\rangle)$.

Here the set $S$ is an independent set. If not then $a_{1}=\alpha a_{2}+\beta a_{3}$ for $\alpha, \beta \in F$.

So,

$a_{1}=\alpha(\langle 0.5,0.3\rangle,\langle 0.5,0.1\rangle,\langle 0.4,0.2\rangle)+$

$\beta(\langle 0.7,0.3\rangle,\langle 0.7,0.2\rangle,\langle 0.9,0.1\rangle)$

$=(\langle\max \{\min (0.5, \alpha), \min (0.7, \beta)\}$,

$\min \{\max (0.3,1-\alpha), \max (0.3,1-\beta)\}\rangle$,

$\langle\max \{\min (0.5, \alpha), \min (0.7, \beta)\}$, $\min \{\max (0.1,1-\alpha), \max (0.2,1-\beta)\}\rangle$, $\langle\max \{\min (0.4, \alpha), \min (0.9, \beta)\}$, $\min \{\max (0.2,1-\alpha), \max (0.1,1-\beta)\}\rangle)$.

It is not possible to find any $\alpha, \beta \in F$ such that the corresponding coefficients on both sides will be equal. That is, $a_{1} \neq \alpha a_{2}+\beta a_{3}$.

Similarly, $a_{2} \neq \alpha a_{1}+\beta a_{3}$ and $a_{3} \neq \alpha a_{2}+\beta a_{1}$. So the set $S$ is independent. 
Let $S_{1}=\left\{a_{1}, a_{2}\right\}$ be a subset of $V_{3}$, where $a_{1}=(\langle 0.7,0.3\rangle,\langle 0.5,0.3\rangle,\langle 0.6,0.3\rangle)$ and $a_{2}=(\langle 0.8,0.2\rangle,\langle 0.5,0.1\rangle,\langle 0.6,0.2\rangle)$.

Here $a_{1}=c a_{2}$ for $c=0.7$. So $S_{1}$ is a dependent set.

\section{Definition 15. (Basis)}

Let $W$ be an Atanassov's intuitionistic fuzzy subspace of $V_{n}$ and $S$ be a subset of $W$ such that the elements of $S$ are independent. If every element of $W$ can be expressed uniquely as a linear combination of the elements of $S$, then $S$ is called a basis of $W$.

\section{Definition 16. (Standard basis)}

A basis $B$ of an AIFVS $W$ is a standard basis if and only if whenever $b_{i}=\sum_{j=1}^{n} a_{i j} b_{j}$ for $b_{i}, b_{j} \in B$ and $a_{i j} \in[0,1]$ then $a_{i i} b_{i}=b_{i}$.

Example 3. Let $S=\left\{a_{1}, a_{2}, a_{3}\right\}$ be a subset of $V_{3}$ given by $a_{1}=(\langle 0.5,0.4\rangle,\langle 0.7,0.2\rangle,\langle 0.6,0.3\rangle)$,

$$
a_{2}=(\langle 0.5,0.3\rangle,\langle 0.6,0.2\rangle,\langle 0.8,0.2\rangle) \text { and }
$$
$a_{3}=(\langle 0.4,0.4\rangle,\langle 0.4,0.3\rangle,\langle 0.8,0.1\rangle)$. Here $S$ is independent set, since $a_{1} \neq c_{1} a_{2}+c_{2} a_{3}$, $a_{2} \neq c_{3} a_{1}+c_{4} a_{3}$ and $a_{3} \neq c_{5} a_{1}+c_{6} a_{2}$. So $\left\{a_{1}, a_{2}, a_{3}\right\}$ is a basis for $\langle S\rangle$. Now this is a standard basis also. For $a_{1}=c_{11} a_{1}+c_{12} a_{2}+c_{13} a_{3}$ holds if $c_{11}=0.8, c_{12}=0.5$ and $c_{13}=0.6$. Also $a_{1}=c_{11} a_{1}$ for $c_{11}=0.8$. Similarly, for $a_{2}$ and $a_{3}$.

\section{Definition 17. (Row space and column space)}

Let $A=\left(\left\langle a_{i j \mu}, a_{i j v}\right\rangle\right) \in F_{m \times n}$ be an AIFM. Then the element $\left\langle a_{i j \mu}, a_{i j \nu}\right\rangle$ is the $i j$ th entry of $A$. $A_{i *}\left(A_{* j}\right)$ denotes the $i$ th row ( $j$ th column) of $A$.

The row space $R(A)$ of $A$ is the subspace of $V_{n}$ generated by the rows $\left\{A_{i *}\right\}$ of $A$. The column space $C(A)$ of $A$ is the subspace of $V_{m}$ generated by the columns $\left\{A_{* j}\right\}$ of $A$.

\section{Generalized Inverse}

In this section, the generalized inverse of an AIFM is investigated.

\section{Definition 18. (Generalized inverse)}

An AIFM $A \in F_{m \times n}$ is said to be regular if there exists another AIFM, $X \in F_{n \times m}$ such that $A X A=A$. In this case, $X$ is called a generalized inverse ( $g$-inverse) of $A$ and it is denoted by $A^{-}$.

The $g$-inverse of an AIFM is not unique that is, an AIFM has many $g$-inverses. The set of all such $g$-inverses of $A$ are denoted by $A\{1\}$.

Theorem 1. Let $A$ be an AIFM whose non-zero rows form a standard basis. If for some intuitionistic fuzzy permutation matrix $P, A$ satisfy the matrix equation $A P A=A$ under the max-min operation, then $A$ is regular.

Proof. Here the non-zero rows of an AIFM $A$ form a standard basis. Let $P A=X$, the rows of $X$ are rearrangement of rows of $A$. Then $X$ is an idempotent AIFM, that is $X^{2}=X$, having same row space as $A$ with the non-zero rows of $X$ form a standard basis also. Since standard basis are unique therefore $A=P X$, for some intuitionistic fuzzy permutation matrix $P$. Then, $A P^{T} A=P X P^{T} P X=P X X=P X=A$, that is, $A P A=A$.

Therefore $A$ is regular.

Definition 19. For an AIFM $A$ of order $m \times n$, an AIFM $G \in F_{n \times m}$ is said to be outer inverse of $A$, if $G A G=G$ and is denoted by $A\{2\}$.

$G$ is said to be $\{1,2\}$ inverse or semi inverse of $A$, if $A G A=A$ and $G A G=G$ and is denoted by $A\{1,2\}$.

The AIFM $G$ is said to be $\{1,3\}$ inverse or least square $g$-inverse of $A$ if, $A G A=A$ and $(A G)^{T}=A G$ and is denoted by $A\{1,3\}$. 
Again $G$ is said to be $\{1,4\}$ inverse or minimum norm $g$-inverse of $A$ if, $A G A=A$ and $(G A)^{T}=G A$ and is denoted by $A\{1,4\}$.

No algorithm is available to find $g$-inverse of AIFM. Here we present a simple algorithm to evaluate $g$-inverse of an AIFM.

\section{Algorithm (To find the $g$-inverse of an AIFM $A$ )}

Step 1: Check whether the non-zero rows of AIFM A form a standard basis or not for the row space of A.

Step 2: If non-zero rows form a standard basis then find some AIFPM $\mathrm{P}$ such that APA=A.

Step 3: Choose an AIFM R such that $\mathrm{RA}=\mathrm{A}$.

Step4: Then PR is a g-inverse of A.

The matrix $P R$ is a $g$-inverse of $A$ since $A(P R) A=A P(R A)=A P A=A$.

The following example demonstrates the above algorithm to compute $g$-inverse of $A$.

Example 4. Let us consider an AIFM

$$
A=\left[\begin{array}{ccc}
\langle 0.5,0.4\rangle & \langle 0.7,0.2\rangle & \langle 0.6,0.3\rangle \\
\langle 0.5,0.3\rangle & \langle 0.6,0.2\rangle & \langle 0.8,0.2\rangle \\
\langle 0.4,0.4\rangle & \langle 0.4,0.3\rangle & \langle 0.8,0.1\rangle
\end{array}\right] \text {. }
$$

The rows of $A$ are independent and they form a standard basis. Since, $R_{i}=\sum_{j=1}^{3} a_{i j} R_{j}$ for $R_{i}, R_{j} \in R$ (row space of $A$ ), $a_{i j} \in[0,1]$ and $a_{i i} R_{i}=R_{i}$, $i=1,2,3$.

For the AIFPM $P=\left[\begin{array}{ccc}\langle 0,1\rangle & \langle 1,0\rangle & \langle 0,1\rangle \\ \langle 1,0\rangle & \langle 0,1\rangle & \langle 0,1\rangle \\ \langle 0,1\rangle & \langle 0,1\rangle & \langle 1,0\rangle\end{array}\right]$,

$A P A=A$ holds.

Now, for the AIFM

$$
R=\left[\begin{array}{ccc}
\langle 0.8,0.2\rangle & \langle 0.5,0.5\rangle & \langle 0.5,0.3\rangle \\
\langle 0.4,0.5\rangle & \langle 0.8,0.1\rangle & \langle 0.6,0.3\rangle \\
\langle 0.3,0.4\rangle & \langle 0.4,0.4\rangle & \langle 0.9,0.1\rangle
\end{array}\right],
$$

$R A=A$ holds .

So the $g$-inverse of $A$ is

$$
P R=\left[\begin{array}{ccc}
\langle 0.4,0.5\rangle & \langle 0.8,0.1\rangle & \langle 0.6,0.3\rangle \\
\langle 0.8,0.2\rangle & \langle 0.5,0.5\rangle & \langle 0.5,0.3\rangle \\
\langle 0.3,0.4\rangle & \langle 0.4,0.4\rangle & \langle 0.9,0.1\rangle
\end{array}\right]=X
$$

(say), which satisfy the relation $A X A=A$.

If each row of an AIFM $B$ can be expressed as a linear combination of the rows of AIFM $A$, then we write $R(B) \subseteq R(A)$. If $R(B) \subseteq R(A)$ and $R(A) \subseteq R(B)$, then we say that $R(A)=R(B)$.

Theorem 2. Let $A, B \in F_{m \times n}$ be two AIFMs. If $A$ is regular then,

(a) $R(B) \subseteq R(A)$ iff $B=B A^{-} A$ for each $A^{-} \in A\{1\}$.

(b) $C(B) \subseteq C(A)$ iff $B=A A^{-} B$ for each $A^{-} \in A\{1\}$.

Proof. (a) Let $R(B) \subseteq R(A)$, then each row of $B$ is a linear combination of the rows of $A$. Hence $B_{i^{*}}=\sum x_{i j} A_{j^{*}}$, where $x_{i j} \in\langle F\rangle$.

That is, $B=X A$ (for some $X \in F_{m}$ )

or, $B=X A A^{-} A$ (since $A=A A^{-} A$ )

or, $B=B A^{-} A$.

$$
\text { Conversely, if } B=B A^{-} A \text {, then }
$$

$B=X A A^{-} A$ (for some $X \in F_{m}$ )

or, $B=X A$. (since $A=A A^{-} A$ )

This implies that $R(B) \subseteq R(A)$.

(b) Let $C(B) \subseteq C(A)$.

Then $B=A Y$ (for some $Y \in F_{n}$ ) or, $B=A A^{-} A Y$. (as $A=A A^{-} A$ )

That is $B=A A^{-} B$.

Conversely, if $B=A A^{-} B$, then $B=A A^{-} A Y$ (for some $Y \in F_{n}$ ) or, $B=A Y \quad\left(\right.$ as $A A^{-} A=A$ ) That is $C(B) \subseteq C(A)$. 
Example 5. Let $A=\left[\begin{array}{ll}\langle 0.6,0.2\rangle & \langle 0.5,0.4\rangle \\ \langle 0.7,0.3\rangle & \langle 0.5,0.4\rangle\end{array}\right]$ and $B=\left[\begin{array}{ll}\langle 0.6,0.3\rangle & \langle 0.5,0.4\rangle \\ \langle 0.6,0.4\rangle & \langle 0.5,0.4\rangle\end{array}\right]$ be two AIFMs.

One of the $g$-inverse of $A$ is

$$
A^{-}=\left[\begin{array}{cc}
\langle 0.6,0.2\rangle & \langle 0.5,0.4\rangle \\
\langle 0.7,0.3\rangle & \langle 0.5,0.4\rangle
\end{array}\right],
$$

for which $B=B A^{-} A$ holds.

Also $B=X A$ for $X=\left[\begin{array}{ll}\langle 0.7,0.3\rangle & \langle 0.6,0.3\rangle \\ \langle 0.6,0.4\rangle & \langle 0.4,0.4\rangle\end{array}\right]$ holds.

So, $R(B) \subseteq R(A)$.

Similarly, the result is true for column space also.

Theorem 3. Let $A \in F_{m \times n}$ be a regular AIFM and $G$ be a g-inverse of $A$. Then

(a) $G^{T} \in A^{T}\{1\}$.

(b) If $P$ and $Q$ are AIFPMs, then $Q^{T} G P^{T} \in P A Q\{1\}$.

(c) $A G$ and $G A$ are idempotent.

Proof. (a) Let $G$ be a g-inverse of $A$.

Then $A G A=A$ holds. Taking transpose on both sides, we get

$$
A^{T} G^{T} A^{T}=A^{T} \text {. }
$$

This implies $G^{T} \in A^{T}\{1\}$.

(b) Since $P$ and $Q$ are AIFPMs, $P$ and $Q$ are invertible and $P^{-1}=P^{T}, Q^{-1}=Q^{T}$.

Now,

$P A Q\left(Q^{T} G P^{T}\right) P A Q=P A\left(Q Q^{T}\right) G\left(P^{T} P\right) A Q$

$=P A G A Q \quad\left(\right.$ as $\left.\quad Q Q^{T}=I, P P^{T}=I\right)$

$=P A Q . \quad$ (as $A G A=A)$.

This implies $Q^{T} G P^{T} \in P A Q\{1\}$

(c) Again, $(A G)(A G)=(A G A) G$

$$
=A G \quad \text { (as } A G A=A) \text {. }
$$

Also $(G A)(G A)=(G A G) A$

$$
=G A \quad(\text { as } G A G=G) .
$$

Thus $A G$ and $G A$ are idempotent.

Example 6. Let us consider the AIFM

$$
A=\left[\begin{array}{cc}
\langle 1,0\rangle & \langle 0.5,0.2\rangle \\
\langle 0.6,0.3\rangle & \langle 0.5,0.3\rangle
\end{array}\right]
$$

and one of its $g$-inverse is

$$
G=\left[\begin{array}{cc}
\langle 1,0\rangle & \langle 0.5,0.3\rangle \\
\langle 0.7,0.2\rangle & \langle 0.4,0.4\rangle
\end{array}\right] \text {. }
$$

Now,

$$
\begin{aligned}
A^{T} G^{T} A^{T} & =\left[\begin{array}{cc}
\langle 1,0\rangle & \langle 0.6,0.3\rangle \\
\langle 0.5,0.2\rangle & \langle 0.5,0.3\rangle
\end{array}\right] \\
& =A^{T} .
\end{aligned}
$$

Thus $G^{T} \in A^{T}\{1\}$.

Let $P=\left[\begin{array}{cc}\langle 1,0\rangle & \langle 0,1\rangle \\ \langle 0,1\rangle & \langle 1,0\rangle\end{array}\right]$ and

$Q=\left[\begin{array}{cc}\langle 0,1\rangle & \langle 1,0\rangle \\ \langle 1,0\rangle & \langle 0,1\rangle\end{array}\right]$.

Now,

$Q^{T} G P^{T}=\left[\begin{array}{cc}\langle 0.7,0.2\rangle & \langle 0.4,0.4\rangle \\ \langle 1,0\rangle & \langle 0.5,0.3\rangle\end{array}\right]$

and

$P A Q=\left[\begin{array}{cc}\langle 0.5,0.2\rangle & \langle 1,0\rangle \\ \langle 0.5,0.3\rangle & \langle 0.6,0.3\rangle\end{array}\right]$.

$P A Q\left(Q^{T} G P^{T}\right) P A Q=\left[\begin{array}{cc}\langle 0.5,0.2\rangle & \langle 1,0\rangle \\ \langle 0.5,0.3\rangle & \langle 0.6,0.3\rangle\end{array}\right]$

$\left[\begin{array}{cc}\langle 0.7,0.2\rangle & \langle 0.4,0.4\rangle \\ \langle 1,0\rangle & \langle 0.5,0.3\rangle\end{array}\right]\left[\begin{array}{cc}\langle 0.5,0.2\rangle & \langle 1,0\rangle \\ \langle 0.5,0.3\rangle & \langle 0.6,0.3\rangle\end{array}\right]$

$=\left[\begin{array}{cc}\langle 0.5,0.2\rangle & \langle 1,0\rangle \\ \langle 0.5,0.3\rangle & \langle 0.6,0.3\rangle\end{array}\right]=P A Q$.

That is, $Q^{T} G P^{T} \in P A Q\{1\}$.

$(A G)^{2}=$ 


$$
\begin{aligned}
& {\left[\begin{array}{cr}
\langle 1,0\rangle & \langle 0.5,0.3\rangle \\
\langle 0.6,0.3\rangle & \langle 0.5,0.3\rangle
\end{array}\right]\left[\begin{array}{cc}
\langle 1,0\rangle & \langle 0.5,0.3\rangle \\
\langle 0.6,0.3\rangle & \langle 0.5,0.3\rangle
\end{array}\right]} \\
& =\left[\begin{array}{cc}
\langle 1,0\rangle & \langle 0.5,0.3\rangle \\
\langle 0.6,0.3\rangle & \langle 0.5,0.3\rangle
\end{array}\right]=A G . \\
& (G A)^{2}= \\
& {\left[\begin{array}{cr}
\langle 1,0\rangle & \langle 0.5,0.2\rangle \\
\langle 0.7,0.2\rangle & \langle 0.5,0.2\rangle
\end{array}\right]\left[\begin{array}{cc}
\langle 1,0\rangle & \langle 0.5,0.2\rangle \\
\langle 0.7,0.2\rangle & \langle 0.5,0.2\rangle
\end{array}\right]} \\
& =\left[\begin{array}{cc}
\langle 1,0\rangle & \langle 0.5,0.2\rangle \\
\langle 0.7,0.2\rangle & \langle 0.5,0.2\rangle
\end{array}\right]=G A .
\end{aligned}
$$

Theorem 4. Let $A$ be an AIFM, $Y, Z \in A\{1\}$ and $X=Y A Z$. Then $X \in A\{1,2\}$, that is, $X$ is a semiinverse of $A$.

Proof. Since $Y, Z \in A\{1\} \Rightarrow A Y A=A$ and $A Z A=A$.

As $X=Y A Z$ so, $A X A=A(Y A Z) A$

$$
\begin{aligned}
& =(A Y A) Z A \\
& =A Z A \\
& =A .
\end{aligned}
$$

Also

$$
\begin{aligned}
& X A X=(Y A Z) A(Y A Z) \\
& =Y(A Z A)(Y A Z) \\
& =Y(A Y A) Z \\
& =Y A Z \\
& =X .
\end{aligned}
$$

So $X$ is a semi-inverse of the AIFM $A$.

Example 7. Let us consider AIFM

$$
\begin{aligned}
& A= .\left[\begin{array}{ccc}
\langle 0.8,0.2\rangle & \langle 0.6,0.3\rangle & \langle 0.4,0.3\rangle \\
\langle 0.5,0.3\rangle & \langle 0.5,0.1\rangle & \langle 0.4,0.2\rangle \\
\langle 0.7,0.3\rangle & \langle 0.7,0.2\rangle & \langle 0.9,0.1\rangle
\end{array}\right] . \\
& \text { Let } Y=\left[\begin{array}{lll}
\langle 0.8,0.1\rangle & \langle 0.5,0.4\rangle & \langle 0.4,0.4\rangle \\
\langle 0.4,0.6\rangle & \langle 0.7,0.1\rangle & \langle 0.3,0.5\rangle \\
\langle 0.6,0.3\rangle & \langle 0.6,0.2\rangle & \langle 0.9,0.1\rangle
\end{array}\right] \text { and } \\
& Z=\left[\begin{array}{ccc}
\langle 0.9,0.1\rangle & \langle 0.6,0.4\rangle & \langle 0.4,0.5\rangle \\
\langle 0.5,0.3\rangle & \langle 0.6,0.1\rangle & \langle 0.4,0.3\rangle \\
\langle 0.5,0.4\rangle & \langle 0.6,0.3\rangle & \langle 1,0\rangle
\end{array}\right]
\end{aligned}
$$

be two of its $g$-inverses of $A$ of type $A\{1\}$.
Then, $X=Y A Z=$

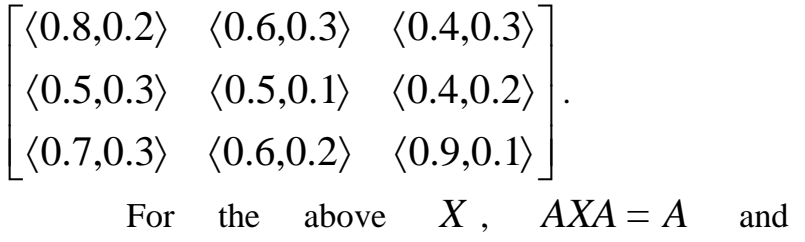
$X A X=X$ holds. So $X$ is a semi-inverse of the AIFM $A$.

Theorem 5. Let $A \in F_{m \times n}$ be an AIFM and $X \in A\{1\}$, then $X \in A\{2\}$ if and only if $R(A X)=R(X)$.

Proof. $X \in A\{2\}$ implies $X A X=A$.

That is, $A \in X\{1\}$.

Hence, $R(X)=R(A X)$ ( since $A X$ is idempotent). Conversely, let $R(A X)=R(A)$, then for a pair of matrices $A$ and $X$, if the product $A X$ is defined so, $R(A X) \subseteq R(X)$.

That is, $X=Y A X$, for some $Y \in F_{m}$.

So $X(A X)=(Y A X) A X$

or, $X A X=Y(A X A) X=Y A X=X$.

Hence $X \in A\{2\}$.

Theorem 6. If $A \in F_{n}$ be a symmetric and idempotent AIFM then $A$ itself a least square $g$-inverse.

Proof. Since $A$ is symmetric, $A^{T}=A$ and $A$ is idempotent, $A^{2}=A$.

Now $P A=A$ if $P=I_{n}$.

Then $A P A=A A=A^{2}=A$.

That is, $A \in A\{1\}$.

Now $(A X)^{T}=X^{T} A^{T}=X^{T} A=A^{T} A$ (Taking

$X=A$, as $A$ itself a $g$-inverse.)

$$
=A A=A X \text {. }
$$

This implies, $A \in A\{1,3\}$.

Theorem 7. If $A \in F_{n}$ be a symmetric and idempotent AIFM then $A$ itself a minimum norm $g$-inverse.

Proof. Here $A^{T}=A$ and $A^{2}=A$.

For $P=I_{n}, P A=A$. 
Then $A P A=A A=A^{2}=A$.

That is, $A \in A\{1\}$.

Now $\quad(X A)^{T}=A^{T} X^{T}=A X^{T}=A A^{T} \quad$ (Taking $X=A$, as $A$ itself a $g$-inverse.)

$$
=A A=X A \text {. }
$$

Hence $A \in A\{1,4\}$.

Example 8. Let us consider the symmetric AIFM

$$
A=\left[\begin{array}{ll}
\langle 0.8,0.2\rangle & \langle 0.6,0.4\rangle \\
\langle 0.6,0.4\rangle & \langle 0.7,0.3\rangle
\end{array}\right]
$$

Now,

$$
\begin{aligned}
A^{2} & =\left[\begin{array}{ll}
\langle 0.8,0.2 & \langle 0.6,0.4 \\
\langle 0.6,0.4\rangle & \langle 0.7,0.3\rangle
\end{array}\right]\left[\begin{array}{ll}
\langle 0.8,0.2 & \langle 0.6,0.4\rangle \\
\langle 0.6,0.4 & \langle 0.7,0.3\rangle
\end{array}\right] \\
& =\left[\begin{array}{ll}
\langle 0.8,0.2\rangle & \langle 0.6,0.4\rangle \\
\langle 0.6,0.4 & \langle 0.7,0.3\rangle
\end{array}\right] \\
& =A .
\end{aligned}
$$

This shows that $A$ is symmetric and idempotent. $A$ satisfy the relation $A X A=A$ for $X=A$, itself.

Again $(A A)^{T}=\left(A^{2}\right)^{T}=A=A A$.

So $A \in A\{1,3\}$ and $A\{1,4\}$.

This shows that $A$ is symmetric and idempotent. $A$ satisfy the relation $A X A=A$ for $X=A$, itself.

Again $(A A)^{T}=\left(A^{2}\right)^{T}=A=A A$.

So $A \in A\{1,3\}$ and $A\{1,4\}$.

Theorem 8. If $A \in F_{n}$ be a symmetric and idempotent AIFM, then $\left\{A+H:\right.$ for all AIFM $H \in F_{n}$ such that $A G \geq A H\}$ is the set of all $\{1,3\}$ inverses of $A$, dominating $A$.

Proof. Since $A$ is symmetric and idempotent AIFM, $A$ itself $A\{1,3\}$ inverse.

Let $\beta$ denote the set $\{A+H$ : for all AIFM $H \in F_{n} \quad$ such that $\left.A G \geq A H\right\}$. Suppose $G \in A\{1,3\}$, then $G \geq A$.

Let $G-A=H$. Since $A\{1,3\} \subseteq A\{1\}$, $G \geq A+H \geq A$.

Implies, $A G \geq A(A+H) \geq A A \geq A$.
Now $G \in A\{1,3\}$ and $A$ itself $A\{1,3\}$ inverse so, as the set $A\{1,3\}$ consists of all solutions for $X$ of $A X=A$ (as $A$ is idempotent).

Thus $A G=A$.

This gives $A(A+H)=A$.

That is $A \geq A H$.

Now, by (1) $A G \geq A H$.

Hence $(A+H) \in \beta$.

Thus for each $G \in A\{1,3\}$ there exists a unique element in $\beta$.

Conversely, for any $G \in \beta, G=A+H \geq A$ with $A \geq A H$.

Hence, $A G=A+A H=A$.

So, $G \in A\{1,3\}$.

Example 9. Consider the AIFM

$$
A=\left[\begin{array}{ll}
\langle 0.8,0.2\rangle & \langle 0.6,0.4\rangle \\
\langle 0.6,0.4\rangle & \langle 0.7,0.3\rangle
\end{array}\right] \text {. }
$$

Here $A^{2}=A$ and $A^{T}=A$.

For the AIFM $G=\left[\begin{array}{ll}\langle 0.9,0.1\rangle & \langle 0.6,0.4\rangle \\ \langle 0.6,0.4\rangle & \langle 0.8,0.2\rangle\end{array}\right]$.

$$
A G A=A \text { and }(A G)^{T}=A G \text {. So }
$$

$G \in A\{1,3\}$.

For the AIFM $H=\left[\begin{array}{ll}\langle 0.7,0.3\rangle & \langle 0.5,0.4\rangle \\ \langle 0.6,0.4\rangle & \langle 0.6,0.4\rangle\end{array}\right]$.

$A G=\left[\begin{array}{cc}\langle 0.8,0.2\rangle & \langle 0.6,0.4\rangle \\ \langle 0.6,0.4\rangle & \langle 0.7,0.3\rangle\end{array}\right]$ and

$A H=\left[\begin{array}{cc}\langle 0.7,0.3\rangle & \langle 0.6,0.4\rangle \\ \langle 0.6,0.4\rangle & \langle 0.6,0.4\rangle\end{array}\right]$.

Note that, $A G \geq A H$.

Then, $\begin{aligned} A+H & =\left[\begin{array}{ll}\langle 0.8,0.2\rangle & \langle 0.6,0.4\rangle \\ \langle 0.6,0.4\rangle & \langle 0.7,0.3\rangle\end{array}\right] \\ & =A \in A\{1,3\} .\end{aligned}$

Theorem 9. If $A \in F_{n}$ be symmetric and idempotent $A I F M$, then $\left\{A+K:\right.$ for all AIFM $K \in F_{n}$ such that 
$A G \geq K A\}$ is the set of all $\{1,4\}$ inverses of $A$, dominating $A$.

Proof. Since $A$ is symmetric and idempotent AIFM, $A$ itself $A\{1,4\}$ inverse.

Let $\beta$ denote the set $\{A+K$ : for all AIFM $K \in F_{n} \quad$ such that $\left.A G \geq K A\right\}$. Suppose $G \in A\{1,4\}$, then $G \geq A$.

Let $G-A=K$. Since $A\{1,4\} \subseteq A\{1\}$, $G \geq A+K \geq A$.

This implies $G A \geq(A+K) A \geq A A \geq A$.

Since $G \in A\{1,4\}$ and $A$ itself $A\{1,4\}$ inverse and the set $A\{1,4\}$ consists of all solutions for $X$ of $X A=A$ (as $A$ is idempotent).

Thus $G A=A$.

This gives $(A+K) A=A$.

That is $A \geq K A$.

Now, by (2) $G A \geq K A$.

Hence $(A+K) \in \beta$.

Thus for each $G \in A\{1,4\}$ there exists a unique element in $\beta$.

Conversely, for any $G \in \beta$,

$G=A+K \geq A$ with $G A \geq K A$.

Hence, $G A=A+K A=A$.

So, $G \in A\{1,4\}$.

Example 10. Consider the AIFM

$$
A=\left[\begin{array}{ll}
\langle 0.7,0.2\rangle & \langle 0.5,0.4\rangle \\
\langle 0.5,0.4\rangle & \langle 0.6,0.3\rangle
\end{array}\right] \text {. }
$$

Here $A^{2}=A$ and $A^{T}=A$.

For the AIFM $G=\left[\begin{array}{cc}\langle 0.8,0.1\rangle & \langle 0.5,0.4\rangle \\ \langle 0.5,0.4\rangle & \langle 0.7,0.2\rangle\end{array}\right]$.

$A G A=A$ and $(G A)^{T}=G A$. So $G \in A\{1,4\}$.

For the AIFM $K=\left[\begin{array}{ll}\langle 0.6,0.3\rangle & \langle 0.5,0.4\rangle \\ \langle 0.5,0.5\rangle & \langle 0.5,0.5\rangle\end{array}\right]$.

$G A=\left[\begin{array}{ll}\langle 0.7,0.2\rangle & \langle 0.5,0.4\rangle \\ \langle 0.5,0.4\rangle & \langle 0.6,0.3\rangle\end{array}\right]$ and

$K A=\left[\begin{array}{ll}\langle 0.6,0.3\rangle & \langle 0.5,0.4\rangle \\ \langle 0.5,0.5\rangle & \langle 0.5,0.5\rangle\end{array}\right]$.
Obviously, $G A \geq K A$.

Then, $A+K=\left[\begin{array}{ll}\langle 0.7,0.2\rangle & \langle 0.5,0.4\rangle \\ \langle 0.5,0.4\rangle & \langle 0.6,0.3\rangle\end{array}\right]$

$$
=A \in A\{1,4\} \text {. }
$$

\section{Moore-Penrose Inverse}

Definition 20. (Moore-Penrose inverse) For an AIFM $A \in F_{m \times n}$, an AIFM $G \in F_{m \times n}$ is said to be a Moore-Penrose inverse of $A$, if $A G A=A, G A G=G,(A G)^{T}=A G$ and $(G A)^{T}=G A$.

The Moore-Penrose inverse of $A$ is denoted by $A^{+}$.

\section{Definition 21. (Minus ordering)}

Let $A$ and $B$ be two AIFMs of order $m \times n$. The minus ordering between $A$ and $B$ is denoted by $A \leq^{-} B$. Then for some $A^{-} \in A\{1\}$ we say $A \leq^{-} B$ if and only if $A A^{-}=B A^{-}$and $A^{-} A=A^{-} B$.

Theorem 10. Let $A, B \in F_{m \times n}$ and $A^{+}$exists, then the following are equivalent.

(a) $A \leq^{-} B$,

(b) $A^{+} A=A^{+} B ; A A^{+}=B A^{+}$,

(c) $A A^{+} B=A=B A^{+} A$.

Proof. $(a) \Rightarrow(b)$

$A \leq^{-} B$ implies $A A^{-}=B A^{-}$and $A^{-} A=A^{-} B$ for some $A^{-} \in A\{1\}$.

Now $A=A A^{-} A=A A^{-} B$ as $A^{-} \in A\{1\}$.

So $A^{+} A=A^{+} A A^{+} B=A^{+} B$.

Similarly, $A A^{+}=B A^{+}$.

$(b) \Rightarrow(c)$

$A^{+} A=A^{+} B$. This gives $A=A A^{+} A=A A^{+} B$.

Also, from $A A^{+}=B A^{+}, A=A A^{+} A=B A^{+} A$.

Thus $A=A A^{+} B=B A^{+} A$.

$(c) \Rightarrow(a)$

Let $X=A^{+} A A^{+}$. 
Then

$A X A=A\left(A^{+} A A^{+}\right) A=\left(A A^{+} A\right) A^{+} A=A A^{+} A=A$.

Thus, $X$ is a g-inverse of $A$.

Now,

$X A=\left(A^{+} A A^{+}\right) A A^{+} B$

$=A^{+}\left(A A^{+} A\right) A^{+} B=\left(A^{+} A A^{+}\right) B=X B$.

Similarly, $A X=B X$.

Hence $A \leq^{-} B$ for $X \in A\{1\}$.

Theorem 11. If $A \leq^{-} B$ and $B$ is idempotent then $B$ is a $g$-inverse of $A$. Also, if $A^{+}$exists then $B$ will be a $g$-inverse of $A^{+}$.

Proof. Since $B$ is idempotent then $B$ is regular and $B$ itself is a g-inverse of $B$. Here $B \in B\{1\}$.

Now $A \leq^{-} B$ implies $A=A A^{-} B=B A^{-} A$.

So,

$A B^{-} A=\left(A A^{-} B\right) B^{-}\left(B A^{-} A\right)$

$=\left(A A^{-} B\right) A^{-} A=A A^{-} A=A$,

for each $B^{-} \in B\{1\}$.

This implies $B\{1\} \subseteq A\{1\}$.

Hence $B$ is a g-inverse of $A$.

Now if $A^{+}$exists then,

$A^{+}=A^{+} A A^{+}=A^{+} B A^{+}$( since $A^{+} A=A^{+} B$ ).

Hence $B \in A^{+}\{1\}$.

$A^{+}=A^{+} A A^{+}=A^{+} B A^{+}$( since $A^{+} A=A^{+} B$ ).

Hence $B$ is a $g$-inverse of $A^{+}$.

Remark 1. The condition on $B$ to be idempotent is essential for $B \in A\{1\}$.

Example 11. Let $A=\left[\begin{array}{cc}\langle 1,0\rangle & \langle 1,0\rangle \\ \langle 0.5,0.5\rangle & \langle 0,1\rangle\end{array}\right]$ and $B=$ $\left[\begin{array}{cc}\langle 1,0\rangle & \langle 1,0\rangle \\ \langle 0.5,0\rangle & \langle 0,1\rangle\end{array}\right]$

Now $B^{2}=\left[\begin{array}{cc}\langle 1,0\rangle & \langle 1,0\rangle \\ \langle 0.5,0\rangle & \langle 0.5,0\rangle\end{array}\right] \neq B$, so $B$ is not idempotent.
Here, $A\{1\}=X: X=\left[\begin{array}{cc}\langle a, b\rangle & \langle c, d\rangle \\ \langle e, f\rangle & \langle g, h\rangle\end{array}\right]$ where

$a=0, b=1 ; c \geq 0.5, d \leq 0.5 ; e=1, f=0$;

$0 \leq g \leq 1,0 \leq h \leq 1$.

Theorem 12. Let $A \in F_{m \times n}$ be a regular AIFM with $Y$ be its minimum norm $g$-inverse and $Z$ be its least square $g$-inverse. Then $Y A Z=A^{+}$, where $A^{+}$is the Moore-Penrose inverse of $A$.

Proof. Let $X=Y A Z$ then as $Y, Z \in A\{1\}$ so, $X \in A\{1,2\}$, that is, $X$ is the semi inverse of $A$. $A X=A Y A Z=A Z$ ( as $A Y A=A$ ).

Now, $(A X)^{T}=(A Z)^{T}=A Z$ ( since $Z \in A\{1,3\}$ ) $=A X$.

Again, $X A=Y A Z A=Y A($ since $A Z A=A)$.

And $(X A)^{T}=(Y A)^{T}=Y A$

(since $Y \in A\{1,4\}$ )

$=X A$.

Thus $X=Y A Z=A^{+}$.

Example 12 Let us consider an AIFM

$$
\begin{aligned}
& A=\left[\begin{array}{ll}
\langle 0.8,0.2\rangle & \langle 0.6,0.4\rangle \\
\langle 0.6,0.4\rangle & \langle 0.7,0.3\rangle
\end{array}\right] \text { and } \\
& Y=\left[\begin{array}{ll}
\langle 0.8,0.2\rangle & \langle 0.6,0.4\rangle \\
\langle 0.5,0.4\rangle & \langle 0.7,0.3\rangle
\end{array}\right], \\
& Z=\left[\begin{array}{ll}
\langle 0.9,0.1\rangle & \langle 0.6,0.4\rangle \\
\langle 0.5,0.5\rangle & \langle 0.7,0.2\rangle
\end{array}\right]
\end{aligned}
$$

be two of its $g$-inverses.

Now, $A Z=\left[\begin{array}{cc}\langle 0.8,0.2\rangle & \langle 0.6,0.4\rangle \\ \langle 0.6,0.4\rangle & \langle 0.7,0.3\rangle\end{array}\right]$ and $(A Z)^{T}=A Z$.

That is, $Z$ is the least square $g$-inverse of $A$.

$Y A=\left[\begin{array}{cc}\langle 0.8,0.2\rangle & \langle 0.6,0.4\rangle \\ \langle 0.6,0.4\rangle & \langle 0.7,0.3\rangle\end{array}\right]$ and $(Y A)^{T}=Y A$.

Therefore, $Y$ is the minimum norm $g$-inverse of $A$.

Now $X=Y A Z=\left[\begin{array}{cc}\langle 0.8,0.2\rangle & \langle 0.6,0.4\rangle \\ \langle 0.6,0.4\rangle & \langle 0.7,0.3\rangle\end{array}\right]$ for

which 
$A X A=A, X A X=X,(A X)^{T}=A X$ and $(X A)^{T}=X A$ holds.

So $X=Y A Z$ is the Moore-Penrose inverse of $A$.

Theorem 13. If $A \in F_{n}$ be a symmetric idempotent

AIFM, then $A^{+}=A$.

Proof. Since $A$ is symmetric and idempotent so $A \in\{1,3\}$ and $A \in\{1,4\}$.

Hence, $A^{+}=A\{1,4\} A A\{1,3\}$ (by theorem 12)

$$
=(A+K) A(A+H) \text { (since } A+H \quad \text { and }
$$

$A+K$ are the set of all $\{1,3\}$ and $\{1,4\}$ inverses respectively)

$$
\begin{aligned}
& =(A+H)\left(A^{2}+A K\right) \\
& =(A+H)(A+A K) \\
& =(A+H) A(\text { since } A \geq A K, A+A K=A) \\
& =A^{2}+H A \\
& =A+H A \\
& =A .(\text { since } A \geq H A, A+H A=A) .
\end{aligned}
$$

Example 13. Let $A=\left[\begin{array}{ll}\langle 0.7,0.2\rangle & \langle 0.5,0.4\rangle \\ \langle 0.5,0.4\rangle & \langle 0.6,0.3\rangle\end{array}\right]$ be an AIFM.

Here $A$ is symmetric and idempotent, that is, $A^{T}=A$ and $A^{2}=A$. Now,

$$
A\{1\}=X: X=\left[\begin{array}{ll}
\langle a, b\rangle & \langle c, d\rangle \\
\langle e, f\rangle & \langle g, h\rangle
\end{array}\right]
$$

where,

$a \geq 0.7, b \leq 0.2$ such that , $a+b \leq 1$

$0 \leq c \leq 1, d \geq 0.4$ such that, $c+d \leq 1$

$e \leq 0.5, f \geq 0.4$ such that, $e+f \leq 1$

$g \geq 0.6, h \geq 0.3$ such that, $g+h \leq 1$.

Here $X=A \in A\{1\}$ as it satisfied the above condition. So, $A X A=A ; X A X=X ;(A X)^{T}=A X$ and $(X A)^{T}=X A$ for $X=A$.

Therefore, $X=A$ is itself a Moore-Penrose inverse, that is $A=A^{+}$.

\section{An Application}

We can used the $g$-inverse of AIFMs to find the solution of Atanassov intuitionistic fuzzy relational equations.

Let us consider the system of Atanassov intuitionistic fuzzy equations $A X=B$ where,

$$
\begin{aligned}
& A=\left[\begin{array}{lll}
\langle 0.7,0.3\rangle & \langle 0.6,0.4\rangle & \langle 0.5,0.5\rangle \\
\langle 0.5,0.5\rangle & \langle 0.6,0.3\rangle & \langle 0.8,0.2\rangle
\end{array}\right], \\
& X=\left[\begin{array}{c}
\left\langle x_{1 \mu}, x_{1 v}\right\rangle \\
\left\langle x_{2 \mu}, x_{2 v}\right\rangle \\
\left\langle x_{3 \mu}, x_{3 v}\right\rangle
\end{array}\right] \text { and } B=\left[\begin{array}{c}
\langle 0.6,0.3\rangle \\
\langle 0.5,0.4\rangle
\end{array}\right] \text {. }
\end{aligned}
$$

Each particular matrix $X$ that satisfy the equation $A X=B$ is called its solution and the set $\Omega(A B)=A^{-} B$ denotes the set of all solutions.

An AIFM may have multiple $g$-inverses. Here we consider one of the generalized inverse of the AIFM $A$, which is $A^{-}=\left[\begin{array}{ll}\langle 0.8,0.2\rangle & \langle 0.5,0.5\rangle \\ \langle 0.5,0.5\rangle & \langle 0.5,0.5\rangle \\ \langle 0.5,0.5\rangle & \langle 0.8,0.2\rangle\end{array}\right]$.

Then, $X \in \Omega(A B)=A^{-} B=\left[\begin{array}{c}\langle 0.6,0.3\rangle \\ \langle 0.5,0.5\rangle \\ \langle 0.5,0.4\rangle\end{array}\right]$, is one of the solution of the above system of equations.

\section{Conclusion}

We introduced the concept of standard basis for AIFVs. Also, a method to find the g-inverse of an AIFM is described. The g-inverse does not exist for all AIFMs. The condition for the existence of the g-inverse are discussed in this paper. A suitable application of ginverse is also provided. More investigation is required for the existence of g-inverse of all AIFMs. If we replace max-min operations by $t$-norm and $t$-conorm, then we obtain another kind of g-inverse of AIFM. In the next paper we try to prove some related properties of g-inverse of AIFM. 


\section{Acknowledgements}

We are very grateful and would like to express our sincere thanks to Editor-in-Chief and anonymous referees for their valuable comments and suggestions which help us to improving this paper a lot.

\section{References}

1. L.A. Zadeh, Fuzzy sets, Information and Control, 8, 338-353 (1965).

2. K.T. Atanassov, Intuitionistic fuzzy sets, Fuzzy Sets and Systems, 20, 87-96 (1986).

3. J. Cen, Fuzzy matrix partial ordering and generalized inverses, Fuzzy Sets and Systems, 15, 453-458 (1999).

4. J. Cen, On generalized inverses of fuzzy matrices, Fuzzy Sets and Systems, 5(1), 66-75 (1991).

5. S.K. Khan and M. Pal, Intuitionistic fuzzy tautological matrices, Journal of Physical Science, 8, 92-100 (2003).

6. A.R. Meenakhi and C. Inbam, The minus partial order in fuzzy matrices, The Journal of Fuzzy Mathematics, 12(3), 695-700 (2004).

7. S.K. Mitra, Matrix partial order throgh generalized inverses: unified theory, Linear Algebra and its Applications, 148, 237-263 (1991).

8. L.J. Xin, Convergence of power of a controllble fuzzy matrices, Fuzzy Sets and System, 45, 313-319 (1992).

9. S.K. Shyamal and M. Pal, Interval-valued fuzzy matrices, The Journal of Fuzzy Mathematics, 14(3), 583-604 (2006).

10. A. K. Shyamal and M. Pal, Two new operators on fuzzy matrices, J. Applied Mathematics and Computng, 15, 91107 (2004).

11. M. Dehghan, M. Ghatee and B. Hashemi, The inverse of a fuzzzy matrix of fuzzy numbers, International Journal of Computer Mathematics, 86(8), 1433-1452 (2009).

12. M. Panigrahi and S. Nanda, Intuitionistic fuzzy relations over intuitionistic fuzzy sets, The Journal of Fuzzy Mathematics, 15(3), 675-688 (2007).

13. M. Pal, Intuitionistic fuzzy determinant, V.U.J. Physical Sciences, 7, 87-93 (2001).

14. M. Pal, S.K. Khan and A.K. Shyamal, Intuitionistic fuzzy matrices, Notes on Intuitionistic Fuzzy Sets, 8(2), 51-62 (2002).

15. A. K. Shyamal and M. Pal, Distance between intuitionistic fuzzy matrices and its applicatins, Natural and Physical Science, 19(1), 39-58 (2005).

16. A. K. Shyamal and M. Pal, Distance between intuitionistic fuzzy matrices, V.U.J. Physical Sciences, 8, 81-91 (2002).

17. S. Sriram and P. Murugadas, On semiring of intuitionistic fuzzy matrices, Applied Mathematical Science, 4(23), 1099-1105 (2010).
18. T. K. Mondal and S. K. Samanta, Generalized intuitionistic fuzzy sets, The Journal of Fuzzy Mathematics, 10(4), 839-862 (2002).

19. M. Bhowmik and M. Pal, Generalized intuitionistic fuzzy matrices, Far-East Journal of Mathematical Sciences, 29(3), 533-554 (2008).

20. M. Bhowmik and M. Pal, Some results on generalized interval-valued intuitionistic fuzzy sets, International Journal of Fuzzy Systems, 14(2), 193-203 (2012).

21. M. Bhowmik and M. Pal, Generalized interval-valued intuitionistic fuzzy sets, The Journal of Fuzzy Mathematics, 18(2), 357-371 (2010).

22. S.K. Khan and A. Pal, The generalized inverse of intuitionistic fuzzy matrix, Journal of Physical Sciences, 11, 62-67 (2007).

23. A.K. Adak, M. Bhowmik and M. Pal, Some properties of generalized intuitionistic fuzzy nilpotent matrices over distributive lattice, Fuzzy Inf. and Eng., 4(4), 371-387 (2012). 